\title{
A NOTE ON MATRICES MAPPING A POSITIVE VECTOR ONTO ITS ELEMENT-WISE INVERSE
}

\author{
SÉBASTIEN LABBÉ
}

\begin{abstract}
For any primitive matrix $M \in \mathbb{R}^{n \times n}$ with positive diagonal entries, we prove the existence and uniqueness of a positive vector $\mathbf{x}=\left(x_{1}, \ldots, x_{n}\right)^{t}$ such that $M \mathbf{x}=\left(\frac{1}{x_{1}}, \ldots, \frac{1}{x_{n}}\right)^{t}$. The contribution of this note is to provide an alternative proof of a result of Brualdi et al. (1966) on the diagonal equivalence of a nonnegative matrix to a stochastic matrix.
\end{abstract}

\section{INTRODUCTION}

In this note, we consider matrices mapping a vector with positive entries onto its element-wise inverse. We prove unicity and existence of such a vector for primitive matrices, that is nonnegative matrices some power of which is positive, with positive diagonal entries. The main result is:

Theorem 1. Let $M \in \mathbb{R}_{\geq 0}^{n \times n}$ be a primitive matrix with positive diagonal entries. Then there exists a unique vector $\mathbf{x}=\left(x_{1}, \ldots, x_{n}\right)^{t}$ with positive entries such that $M \mathbf{x}=$ $\left(\frac{1}{x_{1}}, \ldots, \frac{1}{x_{n}}\right)^{t}$.

It turns out that this question was already answered in 1966 under an equivalent form. In BPS66], it was proved that if $A$ is a nonnegative square matrix with positive diagonal entries, then there exists a unique diagonal matrix $D$ with positive diagonal entries such that $D A D$ is row stochastic (see also [Sin66] who proved it for positive matrices $A$ ). The equivalence is explained below in Lemma 2.

As a consequence, the contribution of this note is to provide an alternative proof of the above result. Unicity for primitive matrices is obtained as a consequence of Perron theorem whereas existence for nonnegative matrices with positive diagonal entries is deduced from the Brouwer fixed-point theorem.

This note is structured as follows. In Section 2, we present an equivalent system of quadratic equations to be solved. In Section 3, we deduce unicity for primitive matrices from Perron theorem. In Section 4, we reduce the question to finding fixed-points of a function and we recall Brouwer and Banach fixed-point theorems in Section 5. In Section 6, we use Brouwer fixed-point theorem to prove existence for nonnegative matrices with positive diagonal entries. In Section 7, we use Banach fixed-point theorem to prove existence and unicity for nonnegative matrices with relatively large enough diagonal entries including matrices which are not primitive (Proposition 12).

Date: October 24, 2018.

2010 Mathematics Subject Classification. Primary 15B48; Secondary 47H10 and 15B51.

Key words and phrases. Primitive matrices and Stochastic matrices and Fixed-Point theorems and Perron theorem. 

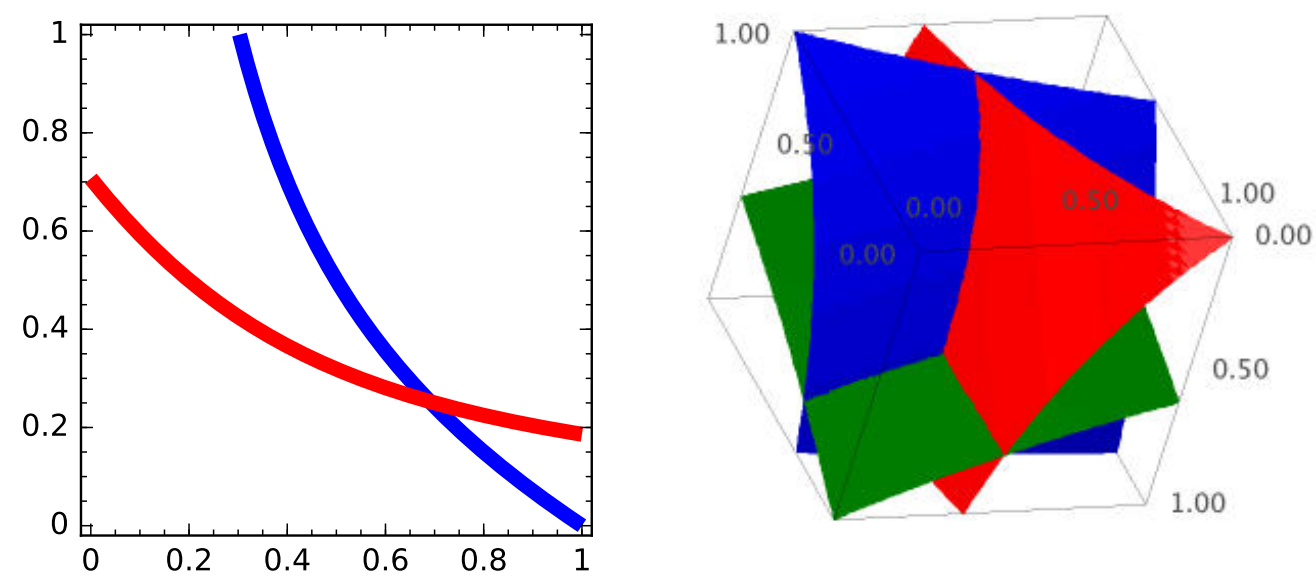

FiguRE 1. Left: the two quadratic curves $x_{1}^{2}+3 x_{1} x_{2}=1,5 x_{1} x_{2}+2 x_{2}^{2}=1$, intersect in a unique point in the box $[0,1]^{2}$. Right: the three quadratic surfaces $x_{1}^{2}+2 x_{1} x_{2}+2 x_{1} x_{3}=1, x_{1} x_{2}+x_{2}^{2}+x_{2} x_{3}=1, x_{1} x_{3}+3 x_{2} x_{3}+x_{3}^{2}=1$ intersect in a unique point in the box $[0,1]^{3}$.

\section{A SYSTEM OF QUADRATIC EQUATIONS}

We say that a vector or a matrix is nonnegative (resp. positive) if all of its entries are nonnegative (resp. positive).

Lemma 2. Let $M=\left(m_{i j}\right) \in \mathbb{R}^{n \times n}$ be a nonnegative matrix and $\mathbf{x}=\left(x_{1}, \ldots, x_{n}\right)^{t}$ be a positive vector. The following conditions are equivalent.

(i) $M \mathbf{x}=\left(\frac{1}{x_{1}}, \ldots, \frac{1}{x_{n}}\right)^{t}$,

(ii) $\operatorname{diag}(\mathbf{x}) M \operatorname{diag}(\mathbf{x})$ is a stochastic matrix,

(iii) for every $i \in\{1, \ldots, n\}$,

$$
x_{i} \sum_{j=1}^{n} m_{i j} x_{j}=1 .
$$

Proof. (i) $\Longleftrightarrow$ (ii). The matrix $\operatorname{diag}(\mathbf{x}) M \operatorname{diag}(\mathbf{x})$ is stochastic if and only if $(1, \ldots, 1)^{t}$ is a right eigenvector with eigenvalue 1 , that is,

$$
\operatorname{diag}(\mathbf{x}) M \operatorname{diag}(\mathbf{x})(1, \ldots, 1)^{t}=(1, \ldots, 1)^{t}
$$

which is equivalent to $M \mathbf{x}=\operatorname{diag}(\mathbf{x})^{-1}(1, \ldots, 1)^{t}=\left(\frac{1}{x_{1}}, \ldots, \frac{1}{x_{n}}\right)^{t}$

(ii) $\Longleftrightarrow$ (iii). Let $\mathbf{r}_{i}$ be the $i$-th row of the matrix $M$. We develop (2) and we get

$$
\operatorname{diag}(\mathbf{x}) M \mathbf{x}=\operatorname{diag}(\mathbf{x})\left(\mathbf{r}_{1} \cdot \mathbf{x}, \ldots, \mathbf{r}_{n} \cdot \mathbf{x}\right)^{t}=\left(x_{1} \mathbf{r}_{1} \cdot \mathbf{x}, \ldots, x_{n} \mathbf{r}_{n} \cdot \mathbf{x}\right)^{t}=(1, \ldots, 1)^{t} .
$$

This equation is verified if and only if, for each $i \in\{1, \ldots, n\}$, the quadratic Equation (1) in $x_{1}, \ldots, x_{n}$ holds.

The system of equations (1) for $i \in\{1, \ldots, n\}$ is illustrated in Figure 1 for $n=2$ and $n=3$. 


\section{UNIQUENESS FOR PRIMITIVE MATRICES}

A primitive matrix is a nonnegative matrix some power of which is positive.

Lemma 3. Let $M \in \mathbb{R}_{\geq 0}^{n \times n}$ be a primitive matrix and $v \in \mathbb{R}_{>0}^{n}$. If $M^{2} v=v$, then $M v=v$.

Proof. We already have that $v$ is a positive vector fixed by $M^{2}$ which is primitive. But so is $M v$ :

$$
M^{2}(M v)=M\left(M^{2} v\right)=M v .
$$

By Perron's theorem, $v$ and $M v$ must be colinear, that is, there exists $\lambda \in \mathbb{R}$ such that $v=\lambda M v$. Then, $v=\lambda^{2} M^{2} v=\lambda^{2} v$ and thus $\lambda^{2}=1$. Since $v$ and $M v$ are positive, we deduce $\lambda=1$.

Proposition 4. Let $M \in \mathbb{R}^{n \times n}$ be a primitive matrix. If there exists a positive vector $\mathbf{x}=\left(x_{1}, \ldots, x_{n}\right)^{t} \in \mathbb{R}_{>0}^{n}$ such that $M \mathbf{x}=\left(\frac{1}{x_{1}}, \ldots, \frac{1}{x_{n}}\right)^{t}$, then it is unique.

Proof. Let $\mathbf{x}=\left(x_{1}, \ldots, x_{n}\right)^{t}, \mathbf{y}=\left(y_{1}, \ldots, y_{n}\right)^{t} \in \mathbb{R}_{>0}^{n}$. Suppose that $X=\operatorname{diag}(\mathbf{x})$ and $Y=\operatorname{diag}(\mathbf{y})$ are such that $X M X$ and $Y M Y$ are both stochastic. The product of diagonal matrices commutes, so we have

$$
(X M Y)^{2}=X M(Y X) M Y=X M(X Y) M Y=(X M X)(Y M Y) .
$$

We conclude that $(X M Y)^{2}$ is stochastic. From Lemma 3 , we conclude that $X M Y$ is stochastic. Thus we have

$$
X M Y(1, \ldots, 1)^{t}=(1, \ldots, 1)^{t} \quad \text { and } \quad Y M Y(1, \ldots, 1)^{t}=(1, \ldots, 1)^{t}
$$

and

$$
\left(x_{1}^{-1}, \ldots, x_{n}^{-1}\right)=X^{-1}(1, \ldots, 1)^{t}=M Y(1, \ldots, 1)^{t}=Y^{-1}(1, \ldots, 1)^{t}=\left(y_{1}^{-1}, \ldots, y_{n}^{-1}\right) .
$$

Therefore $\mathbf{x}=\mathbf{y}$. The conclusion follows from Lemma 2 .

\section{Solutions ARE FIXED POINTS}

It can be seen in Figure 1 that the surfaces of each equation in the positive octant are functions of the form $y=f(x)$ or $z=f(x, y)$. We now formalize and prove this.

Let $M=\left(m_{i j}\right) \in \mathbb{R}^{n \times n}$ be a nonnegative matrix. For each $i \in\{1, \ldots, n\}$ and $\left(x_{1}, \ldots, x_{i-1}, x_{i+1}, \ldots, x_{n}\right) \in \mathbb{R}_{\geq 0}^{n-1}$, we denote

$$
b_{i}=\sum_{j \neq i} m_{i j} x_{j}
$$

For each $i \in\{1, \ldots, n\}$, we define a function $f_{i}^{(M)}: \mathbb{R}_{\geq 0}^{n-1} \rightarrow \mathbb{R}_{>0}$ :

$$
f_{i}^{(M)}\left(x_{1}, \ldots, x_{i-1}, x_{i+1}, \ldots, x_{n}\right)= \begin{cases}b_{i}^{-1} & \text { if } m_{i i}=0 \\ \frac{-b_{i}+\sqrt{b_{i}^{2}+4 m_{i i}}}{2 m_{i i}} & \text { if } m_{i i} \neq 0 .\end{cases}
$$

Lemma 5. Let $M=\left(m_{i j}\right) \in \mathbb{R}^{n \times n}$ be a nonnegative real matrix, $i \in\{1, \ldots, n\}$ and assume $\mathbf{x}=\left(x_{1}, \ldots, x_{n}\right)^{t} \in \mathbb{R}_{>0}^{n}$. The vector $\mathbf{x}$ satisfies Equation (1) if and only if $x_{i}=f_{i}^{(M)}\left(x_{1}, \ldots, x_{i-1}, x_{i+1}, \ldots, x_{n}\right)$. 
Proof. Equation (1) can be seen as a quadratic equation of the variable $x_{i}$ :

$$
m_{i i} x_{i}^{2}+b_{i} x_{i}-1=0
$$

where $b_{i}=\sum_{j \neq i} m_{i j} x_{j}$ is the coefficient of $x_{i}$ in this quadratic polynomial. If $m_{i i}=0$, then $b_{i} x_{i}=1$ and there is only one solution $x_{i}=b_{i}^{-1}$ to Equation (5). Moreover $x_{i}>0$. If $m_{i i} \neq 0$, then there are exactly two real solutions

$$
\frac{-b_{i}-\sqrt{b_{i}^{2}+4 m_{i i}}}{2 m_{i i}}<0 \quad \text { and } \quad \frac{-b_{i}+\sqrt{b_{i}^{2}+4 m_{i i}}}{2 m_{i i}}>0
$$

to Equation (5), the second one being positive.

For every matrix $M \in \mathbb{R}^{n \times n}$, let

$$
F^{(M)}: \mathbb{R}_{\geq 0}^{n} \rightarrow \mathbb{R}_{>0}^{n}: \mathbf{x} \mapsto\left(\begin{array}{l}
f_{1}^{(M)}\left(x_{2}, x_{3}, \ldots, x_{n}\right) \\
f_{2}^{(M)}\left(x_{1}, x_{3}, \ldots, x_{n}\right) \\
\cdots \\
f_{n}^{(M)}\left(x_{1}, x_{2}, \ldots, x_{n-1}\right)
\end{array}\right)
$$

We now have a new equivalent statement.

Lemma 6. Let $M \in \mathbb{R}^{n \times n}$ be a nonnegative matrix and $\mathbf{x}=\left(x_{1}, \ldots, x_{n}\right)^{t}$ be a positive vector. Then $M \mathbf{x}=\left(\frac{1}{x_{1}}, \ldots, \frac{1}{x_{n}}\right)^{t}$ if and only if $\mathbf{x}$ is a fixed point of $F^{(M)}$.

Proof. Let $\mathbf{x}=\left(x_{1}, \ldots, x_{n}\right)^{t}>0$. We have that $F^{(M)}(\mathbf{x})=\mathbf{x}$ if and only if

$$
x_{i}=f_{i}^{(M)}\left(x_{1}, \ldots, x_{i-1}, x_{i+1}, \ldots, x_{n}\right)
$$

for every $i \in\{1, \ldots, n\}$ if and only if $\mathbf{x}$ satisfies Equation (1) for every $i \in\{1, \ldots, n\}$ from Lemma 5 if and only if $M \mathbf{x}=\left(\frac{1}{x_{1}}, \ldots, \frac{1}{x_{n}}\right)^{t}$ from Lemma 2 .

\section{Fixed-Point theOrems}

From [Zei86], we recall some classical fixed-point theorems.

Theorem 7 (Brouwer Fixed Point Theorem). Every continuous function from a closed ball of a Euclidean space into itself has a fixed point.

We consider closed balls for the $\infty$-norm. For every $\mathbf{a}=\left(a_{1}, \ldots, a_{n}\right), \mathbf{b}=\left(b_{1}, \ldots, b_{n}\right) \in$ $\mathbb{R}^{n}$, the closed ball with center $(\mathbf{a}+\mathbf{b}) / 2$ and radius $\max \left\{0,\left(a_{i}-b_{i}\right) / 2\right\}$ for every $i$-th coordinate, $1 \leq i \leq n$, is denoted by

$$
\operatorname{Box}(\mathbf{a}, \mathbf{b})=\left\{\left(x_{1}, \ldots, x_{n}\right) \in \mathbb{R}^{n} \mid a_{i} \leq x_{i} \leq b_{i}, 1 \leq i \leq n\right\}
$$

A function $F: \mathbb{R}_{\geq 0}^{n} \rightarrow \mathbb{R}_{\geq 0}^{n}$ is said decreasing if $F(\mathbf{x}+\mathbf{t}) \leq F(\mathbf{x})$ for every $\mathbf{x}, \mathbf{t} \in \mathbb{R}_{\geq 0}^{n}$.

Corollary 8. Let $F: \mathbb{R}_{\geq 0}^{n} \rightarrow \mathbb{R}_{\geq 0}^{n}$ be a continuous and decreasing function. Then there exists a vector $\mathbf{x} \in \mathbb{R}_{\geq 0}^{n}$ such that $\mathbf{x}=F(\mathbf{x})$.

Proof. Since $F$ is continuous and decreasing, we have that, for every $\mathbf{a}, \mathbf{b} \in \mathbb{R}_{\geq 0}^{n}$,

$$
F(\operatorname{Box}(\mathbf{a}, \mathbf{b})) \subseteq B o x(F(\mathbf{b}), F(\mathbf{a})) .
$$


Moreover, $F$ reaches its maximal value at $\mathbf{a}=\mathbf{0}$ so that $F\left(\mathbb{R}_{\geq 0}^{n}\right) \subseteq \operatorname{Box}(\mathbf{0}, F(\mathbf{0}))$. Then

$$
F(B o x(\mathbf{0}, F(\mathbf{0}))) \subseteq B o x\left(F^{2}(\mathbf{0}), F(\mathbf{0})\right) \subseteq B o x(\mathbf{0}, F(\mathbf{0}))
$$

and Brouwer fixed point theorem applies since $\operatorname{Box}(\mathbf{0}, F(\mathbf{0}))$ is a closed ball.

5.1. Banach Fixed-Point Theorem. Let $(X, d)$ be a metric space. Then a map $T: X \rightarrow X$ is called a contraction mapping on $X$ if there exists $q \in[0,1)$ such that

$$
d(T(x), T(y)) \leq q d(x, y)
$$

for all $x, y \in X$.

Theorem 9 (Banach Fixed Point Theorem). Let $(X, d)$ be a non-empty complete metric space with a contraction mapping $T: X \rightarrow X$. Then $T$ admits a unique fixed-point $\mathbf{x}$ in $X$.

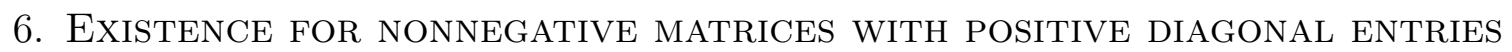

Now we compute the gradient of $f_{i}^{(M)}$ :

$$
\vec{\nabla} f_{i}^{(M)}(\mathbf{x})= \begin{cases}-b_{i}^{-2}\left(m_{i 1}, \ldots, m_{i, i-1}, m_{i, i+1}, \ldots, m_{i n}\right)^{t} & \text { if } m_{i i}=0 \\ \frac{1}{2 m_{i i}}\left(\frac{b_{i}}{\sqrt{b_{i}^{2}+4 m_{i i}}}-1\right)\left(m_{i 1}, \ldots, m_{i, i-1}, m_{i, i+1}, \ldots, m_{i n}\right)^{t} & \text { if } m_{i i} \neq 0\end{cases}
$$

and we conclude that $\vec{\nabla} f_{i}^{(M)} \leq 0$.

We now prove the existence of a fixed point of $F^{(M)}$ using Brouwer fixed-point theorem.

Proposition 10. Let $M=\left(m_{i j}\right) \in \mathbb{R}^{n \times n}$ be a nonnegative real matrix such that $m_{i i}>0$ for every $i$ with $1 \leq i \leq n$. Then there is a positive vector $\mathbf{x}=\left(x_{1}, \ldots, x_{n}\right)^{t}>0$ such that $M \mathbf{x}=\left(\frac{1}{x_{1}}, \ldots, \frac{1}{x_{n}}\right)^{t}$.

Proof. The function $F^{(M)}: \mathbb{R}_{\geq 0}^{n} \rightarrow \mathbb{R}_{>0}^{n}$ is continuous since $m_{i i} \neq 0$ for all $i$ such that $1 \leq i \leq n$. It is decreasing since the entries of its gradient are zero or negative. Thus, Corollary 8 applies and there exists a vector $\mathbf{x} \in \mathbb{R}_{\geq 0}^{n}$ such that $\mathbf{x}=F^{(M)}(\mathbf{x})$. From the definition of $F^{(M)}$, we conclude that the entries of $\mathbf{x}$ are positive, i.e., $\mathbf{x} \in \mathbb{R}_{>0}^{n}$. From Lemma 6, we conclude the existence of a positive vector $\mathbf{x}=\left(x_{1}, \ldots, x_{n}\right)^{t}$ such that $M \mathbf{x}=\left(\frac{1}{x_{1}}, \ldots, \frac{1}{x_{n}}\right)^{t}$.

Example 11. Let

$$
M=\left(\begin{array}{lll}
1 & 0 & 0 \\
1 & 1 & 0 \\
1 & 1 & 1
\end{array}\right) \quad \text { and } \quad \mathbf{x}=\left(1, \frac{\sqrt{5}-1}{2}, \frac{\sqrt{2 \sqrt{5}+22}-\sqrt{5}-1}{4}\right)^{t} .
$$

We verify that

$$
M \mathrm{x}=\left(1, \frac{\sqrt{5}+1}{2}, \frac{\sqrt{5}+\sqrt{2 \sqrt{5}+22}+1}{4}\right)^{t}=\left(1, \frac{2}{\sqrt{5}-1}, \frac{4}{\sqrt{2 \sqrt{5}+22}-\sqrt{5}-1}\right)^{t} .
$$


Proof of Theorem 1. Unicity follows from Proposition 4 since $M$ is primitive. Existence follows from Proposition 10 since diagonal entries of $M$ are positive.

Proposition 10 does not include primitive matrices with zero entries on the diagonal since we can't apply Brouwer fixed-point theorem when $m_{i i}=0$ for some $i$ : $f_{i}^{(M)}$ is not continuous at $\mathbf{0}$ in this case. But the result still holds (see [BPS66, Theorem 8.2]). For example, let

$$
M=\left(\begin{array}{ccc}
0 & 0 & 1 \\
1 & 0 & 0 \\
0 & 1 & 1
\end{array}\right) \quad \text { and } \quad \mathbf{x}=\left(\sqrt{2}, \frac{1}{\sqrt{2}}, \frac{1}{\sqrt{2}}\right)^{t}
$$

We verify that $M \mathbf{x}=\left(\frac{1}{\sqrt{2}}, \sqrt{2}, \sqrt{2}\right)^{t}$.

\section{UNIQUENESS WHEN DIAGONAL ENTRIES ARE RELATIVELY LARGE}

To prove uniqueness in some cases including matrix $M$ from Example 11 which is not primitive, we can use Banach fixed-point theorem. Note that it is not possible to prove that the map $F^{(M)}$ is a contraction for every nonnegative matrix $M$. For example, consider

$$
M=\left(\begin{array}{rrr}
1 & 2 m & 2 m \\
2 m & 1 & 2 m \\
2 m & 2 m & 1
\end{array}\right)
$$

for some $m>0$. We get that the gradient of $F^{(M)}$ at $\mathbf{x}=\mathbf{0}$ (in which case $b_{i}=0$ in Equation (60) is

$$
\left(\frac{\partial F^{(M)}}{\partial x_{i}}(\mathbf{0})\right)_{i=1,2,3}=\left(\begin{array}{rrr}
0 & -m & -m \\
-m & 0 & -m \\
-m & -m & 0
\end{array}\right)
$$

which can get as large as $m$ is. For some matrices $M$, the map $F^{(M)}$ is a contraction as we show now.

Proposition 12. Let $M=\left(m_{i j}\right) \in \mathbb{R}^{n \times n}$ be a nonnegative real matrix such that $2 m_{i i}>$ $m_{i j}$ for every $i, j$ with $1 \leq i, j \leq n$. Then there exists a unique positive vector $\mathbf{x}=$ $\left(x_{1}, \ldots, x_{n}\right)^{t}>0$ such that $M \mathbf{x}=\left(\frac{1}{x_{1}}, \ldots, \frac{1}{x_{n}}\right)^{t}$.

Proof. Existence follows from Proposition 10.

To prove uniqueness we use the Banach Fixed Point Theorem and we show that $F^{(M)}$ is a contraction. From the hypothesis, there exists a constant $C>0$ such that

$$
0 \leq \frac{m_{i j}}{2 m_{i i}} \leq C<1
$$

for every $i, j$ with $1 \leq i, j \leq n$. Thus from Equation (6) and since

$$
\left|\frac{b_{i}}{\sqrt{b_{i}^{2}+4 m_{i i}}}-1\right| \leq 1
$$

we get that

$$
\left\|\vec{\nabla} f_{i}^{(M)}(\mathbf{x})\right\|_{\infty} \leq \frac{1}{2 m_{i i}}\left\|\left(m_{i 1}, \ldots, m_{i, i-1}, m_{i, i+1}, \ldots, m_{i n}\right)^{t}\right\|_{\infty} \leq C
$$


for every $\mathbf{x} \in \mathbb{R}_{\geq 0}^{n-1}$ and $1 \leq i \leq n$. The function $f_{i}^{(M)}$ is differentiable on $\mathbb{R}_{\geq 0}^{n-1}$. Using the Mean value theorem in several variables, for every $\mathbf{a}, \mathbf{b} \in \mathbb{R}_{\geq 0}^{n-1}$ there exists $c \in[0,1]$ such that

$$
f_{i}^{(M)}(\mathbf{b})-f_{i}^{(M)}(\mathbf{a})=\nabla f_{i}^{(M)}((1-c) \mathbf{a}+c \mathbf{b}) \cdot(\mathbf{b}-\mathbf{a}) .
$$

Therefore, by the Cauchy-Schwarz inequality $(|\mathbf{x} \cdot \mathbf{y}| \leq\|\mathbf{x}\|\|\mathbf{y}\|)$,

$$
\left|f_{i}^{(M)}(\mathbf{b})-f_{i}^{(M)}(\mathbf{a})\right| \leq C\|\mathbf{b}-\mathbf{a}\|_{\infty} .
$$

Thus $f_{i}^{(M)}$ is a contraction for every $i$ with $1 \leq i \leq n$. Then $F^{(M)}$ is a contraction. The conclusion is deduced from Lemma 6 .

Proposition 12 seems to hold when $2 m_{i i} \leq m_{i j}$. A possible option in this case is to show that some power of $F^{(M)}$ is a contraction and use a stronger version of Banach theorem: if some iterate $T^{n}$ of $T$ is a contraction, then $T$ has a unique fixed point. More work has to be done.

\section{ACKNOWLEDGEMENTS}

I am thankful to Daniel S. Maynard for fruitful exchanges. The question came up in his research on interference competition in fungi [MBL $\left.{ }^{+} 17\right]$ exploring how spatial structure and species diversity interactively structure the community.

\section{REFERENCES}

[BPS66] Richard A. Brualdi, Seymour V. Parter, and Hans Schneider. The diagonal equivalence of a nonnegative matrix to a stochastic matrix. Journal of Mathematical Analysis and Applications, 16(1):31-50, 1966.

$\left[\mathrm{MBL}^{+} 17\right]$ Daniel S. Maynard, Mark A. Bradford, Daniel L. Lindner, Linda T. A. van Diepen, Serita D. Frey, Jessie A. Glaeser, and Thomas W. Crowther. Diversity begets diversity in competition for space. Nature Ecology $\&$ Evolution, May 2017. http://dx.doi.org/10.1038/ s41559-017-0156.

[Sin66] Richard Sinkhorn. A relationship between arbitrary positive matrices and stochastic matrices. Canadian Journal of Mathematics, 18(0):303-306, January 1966.

[Zei86] Eberhard Zeidler. Nonlinear functional analysis and its applications. I. Springer-Verlag, New York, 1986. Fixed-point theorems, Translated from the German by Peter R. Wadsack.

(S. Labbé) CNRS, LaBRI, UMR 5800, F-33400 Talence, France

E-mail address: sebastien.labbe@labri.fr

$U R L:$ http://www.slabbe.org/ 